\title{
Practical Research Yields Fundamental Insight, Too
}

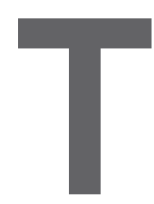

IM WU'S VIEWPoint "Bell Labs and Centralized Innovation" (May 2011) was inaccurate regarding a specific example of research at Bell

Labs.

Wu wrote, "Bell's scientists did cutting-edge work in fields as diverse as quantum physics and data theory. It was a Bell Labs employee named Clinton Davisson who would win a Nobel Prize for demonstrating the wave nature of matter, an insight more typically credited to Einstein than to a telephone company employee." However, Albert Einstein actually discovered that some perplexing data regarding the photoelectric effect could be explained through a hypothesis proposing that light, previously described purely as waves, could behave as particles, now called photons. Others, in particular Louis de Broglie, proposed that matter, previously viewed as particles, could be described by waves. While the Davisson-Germer experiment confirmed de Broglie, neither Davisson nor Lester Germer at the time knew about de Broglie's research; see http://courses. science.fau.edu/voss/modphys/pdf/ Ch05_2.pdf.

Germer (a casual acquaintance) told me he and Davisson did not realize the data showed the wave nature of matter initially due to the wave nature of matter being a rather esoteric idea at the time. That is, they discovered something very important but somewhat by accident. It took time before these two researchers realized what they had actually measured.

There were practical reasons (of interest to a telephone company) for Davisson's and Germer's research, including vacuum tubes, which were then used in amplifiers. Electrons arrive at a vacuum tube's anode with enough energy to cause secondary emission of electrons at the anode, in some cases degrading a vacuum tube's performance.

Understanding how electrons inter- act with an anode was obviously useful in any attempt to improve the anode's design.

William Zaumen, Palo Alto, CA

\section{Author's Response:}

Zaumen is correct. Davisson demonstrated that all particles, not light, have wave-like properties; for example, electrons, and even people, have a wave-like nature. Zaumen is also correct in saying that Einstein worked in a field that assumed light was wave-like, showing its particle-like properties.

Tim Wu, New York

\section{No Reconciling Irreconcilable Models}

Erik Meijer's and Gavin Bierman's article "A Co-Relational Model of Data for Large Shared Data Banks” (Apr. 2011) overreached by claiming equivalence between the Relational Model and NoSQL "key-value pairs" without regard to the definition of a data model by E.F. Codd more than 30 years ago. Finding similarity in NoSQL systems to some parts of the Relational Model, Meijer and Bierman mistakenly concluded the two are equivalent.

Codd, in his paper "Data Models in Database Management" in Proceedings of the 1980 Workshop on Data Abstraction, Databases and Conceptual Modeling (http://portal.acm.org/citation. cfm?id=806891) defined a data model as comprising three components: data structures to represent well-formed expressions in first-order logic; operators closed over these structures, permitting inferencing; and integrity constraints to enforce internal consistency.

NoSQL systems have no data model so defined. All else is commentary.

Meijer and Bierman ignored logic and inferencing and did not explain how key-value systems recognize, let alone enforce, integrity constraints. They cited referential integrity-a form of integrity constraint-as an ex- ogenous cost relational databases bear to correct for a deficiency. The truth is actually the opposite; consistency is a central obligation of any databasemanagement system. The lack of constraint-checking in key-value systems imposes the constraint-checking burden on the application, a situation the Relational Model was invented specifically to correct.

Codd encountered a similar lack of understanding in his day. In the same proceedings paper, he wrote, "In comparing data models people often ignore the operators and integrity rules altogether. When this occurs, the resulting comparisons run the risk of being meaningless."

Codd's landmark article "A Relational Model of Data for Large Shared Data Banks" (Communications, June 1970) addressed other points raised by Meijer and Bierman, including path independence. An interested reader would learn much in an evening spent with that one article alone.

Object Relational Mapping libraries and NoSQL systems attempt to solve (through technical means) a nontechnical problem: reluctance of talented people to master the Relational Model, and thus benefit from its data consistency and logical inferencing capabilities. Rather than exploit it and demand more relational functionality from DBMS vendors, they seek to avoid and replace it, unwittingly advocating a return to the fragile, unreliable, illogical systems of the 1960s, minus the greenbar fanfold paper.

James K. Lowden, New York

\section{Authors' Response:}

Lowden's comment contains a number of errors. Our article was, in fact, explicitly critical of the lack of an agreed data model for NoSQL. We didn't ignore "inferencing," proposing instead a query language based on monad comprehensions-interestingly, the same query language we prefer for the relational model. We did not assert that 
the relational and key-value models are equivalent, but rather dual. The issue of weakening consistency checking goes to the heart of the interest in NoSQL systems and is beyond the scope of our article.

Erik Meijer, Redmond, WA

Gavin Bierman, Cambridge, U.K.

\section{Financial Incentives vs.}

\section{Algorithms in Social Networks}

I thank John C. Tang et al. for their analysis of the crowdsourcing strategies of three successful teams in their article "Reflecting on the DARPA Red Balloon Challenge" (Apr. 2011). Though the iSchools team might have had better data-mining algorithms, it was the MIT team that recognized and exploited financial incentives as the most effective way to be first to identify the 10 red balloons DARPA scattered across the U.S. last year.

In retrospect, the recursive incentive strategy adopted by the MIT team is used in many network-marketing situations worldwide. I first came across it almost 20 years ago when trying to sell a database management system to one of India's oldest non-banking finance companies, which happened to employ a motivated network of insurance agents throughout India. These agents were required to recruit other agents, with the initial premium for the first few months from each new account they signed up distributed hierarchically, though not in the precise geometric progression the MIT team used in the DARPA Challenge. This way, the company's senior agents, having recruited a large network, could virtually sit back and watch as the money poured in. I suppose this, too, is how most Ponzi schemes work, though, in this case, nothing illegal was involved, as is generally implied by the term.

The important takeaway from the Tang et al. analysis is that motivating people is the key to success and that money is often the most effective motivation in any given social network. Whether that is good or bad is a question that needs a totally different kind of analysis.

Prithwis Mukerjee, Kharagpur, India

\section{Let Leap Seconds Sync}

Poul-Henning Kamp's article "The
One-Second War" (May 2011) was enlightening and, from the perspective of an old-time (ex)hardware engineer, entertaining. The reason solder jockeys (hardware engineers) don't see leap seconds as a problem is they presume computers know only what they've been told; if the system clock slows by $1 / 86,400$ th of a second per second, the system's software won't have the slightest idea it happened, nor will it care.

By extension, astronomers using terrestrial time are (by definition) off by some indeterminate amount until leap time, then off in another direction after the leap. Garden-variety system clocks (not directly atomically controlled) are constantly in need of adjustment and aren't very accurate over days at a time. Diddling a fraction of a millisecond out of a second only disappears in the noise. Since atomic clocks are the reference standard, they can skip however many beats are needed to ensure the seconds counter always reads 86,400 when the solar year ends.

Why not make the (invisible to code) system clock adjustable so it always counts to 86,400 seconds until the moment the year counter ticks over? To the code, a second is whatever a register says it is. Hardware, not software, counts electrical oscillations, and if it includes an "add $x$ seconds in $y$ years" pair of adjustment thumbwheels, the result is that 86,400 will have gone by exactly when the (real) year turns over.

Adjusting to leap seconds can be simple, unless programmers try turning a timing-gate issue into a planetary software project. Let astronomers use whatever time-sync definition they want, but if system clocks are adjusted in tiny amounts to keep "better" time, telescopes will be more accurate than if they were abruptly forced to catch up by a full second each year.

Just tell the electrical engineers the numbers and let them provide them to astronomers, system administrators, home users, and everyone else.

David Byrd, Arlington, VA

\section{Author's Response:}

Byrd proposes a number of additional ways we might paper over the fact that the planet is itself an unpredictable and unstable clock. There is no shortage of such ideas, and all are bad hacks. If computers were still huge boxes with a few attached terminals and printer, all these ideas would work, as indeed a number of them did, from the invention of the computer to the mid-1980s. Like today's deployed bad hack-leap seconds-all the schemes Byrd proposes rely on somebody measuring what the planet does and everybody else reacting to it on short notice. His ideas do not improve the current situation in any way but do reintroduce at least one bad idea already discarded-variable-length seconds.

\section{Poul-Henning Kamp,}

Slagelse, Denmark Communications welcomes your opinion. To submit a
Letter to the Editor, please limit yourself to 500 words or less, and send to letters@cacm.acm.org.

\section{(C) 2011 ACM $0001-0782 / 11 / 07 \$ 10.00$}

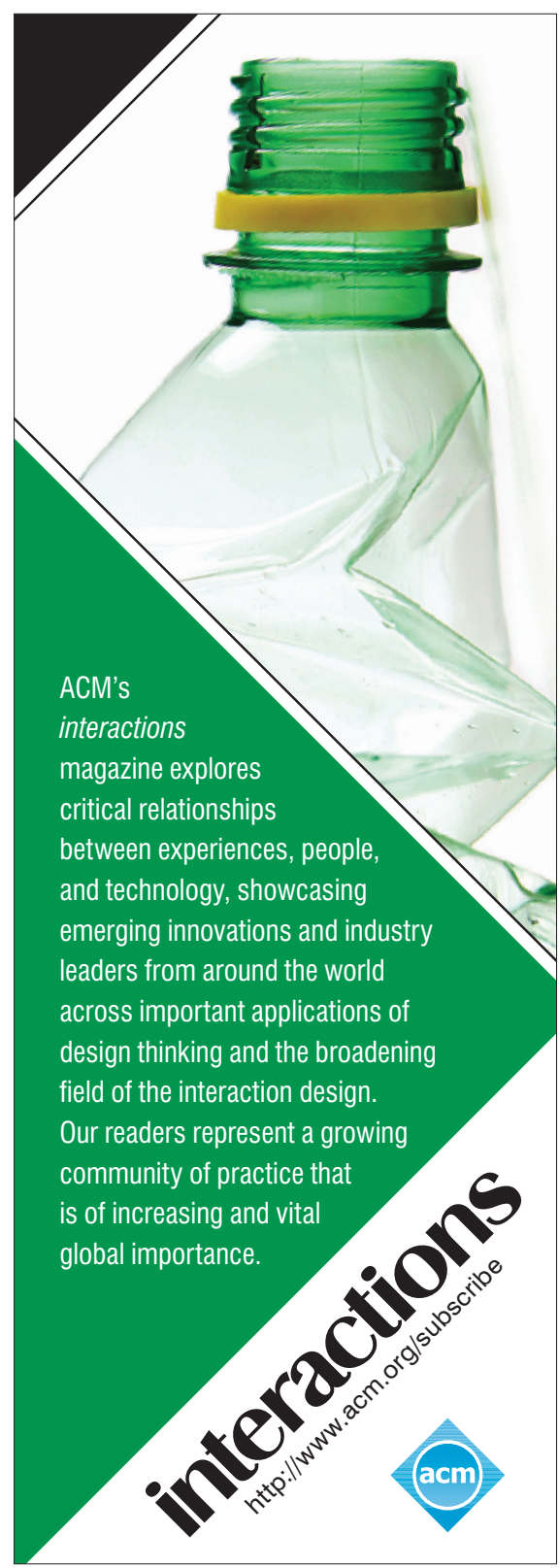

